\title{
Revisión de Megalachne Steud. (Poaceae: Pooideae: Poeae), género endémico del Archipiélago de Juan Fernández, Chile
}

\section{Revision of Megalachne Steud. (Poaceae: Pooideae: Poeae), an endemic genus of Juan Fernandez Archipelago, Chile}

\author{
Carol M. Peña $1,2^{*}$, María A. Negritto ${ }^{3}$, Eduardo Ruiz ${ }^{2}$, Carlos M. Baeza ${ }^{2} \&$ Victor L. Finot $^{4}$ \\ 1Departamento de Ciencia y Tecnología Vegetal, Universidad de Concepción, Campus Los Ángeles, Juan Antonio Coloma \\ 0201, Los Ángeles, Chile. \\ 2Departamento de Botánica, Facultad de Ciencias Naturales y Oceanográficas, Universidad de Concepción, Casilla 160-C, \\ Concepción, Chile. \\ ${ }^{3}$ Universidad del Magdalena, Cra. 32 N²2-08, Santa Marta, Colombia. \\ ${ }^{4}$ Departamento de Producción Animal, Facultad de Agronomía, Universidad de Concepción, Casilla 537, Chillán, Chile. \\ *cpena@udec.cl
}

\begin{abstract}
RESUMEN
Megalachne Steud. (Poaceae: Pooideae: Poeae) es un género endémico del Archipiélago de Juan Fernández, Chile, para el cual se conoce hasta ahora sólo dos especies: M. berteroniana Steud. y M. masafuerana (Skottsb. \& Pilg.) Matthei. Durante la revisión del género se determinó la existencia de una nueva especie, $M$. robinsoniana nov. sp. Se entregan descripciones, ilustraciones, material estudiado y una clave para distinguir las especies.
\end{abstract}

Palabras claVe: Chile, Poaceae, Megalachne robinsoniana, nueva especie.

\begin{abstract}
Megalachne Steud. (Poaceae: Pooideae: Poeae) is an endemic genus from the Juan Fernandez Island, Chile, with two species recognized: M. berteroniana Steud. and M. masafuerana (Skottsb. \& Pilg.) Matthei. During the taxonomic revision of Megalachne a new species was discovered: M. robinsoniana nov. sp. Species are described, illustrated, studied material and a key to distinguish them is given.
\end{abstract}

KeYwords: Chile, Poaceae, Megalachne robinsoniana, new species.

\section{INTRODUCCIÓN}

El Archipiélago de Juan Fernández, ubicado en el Océano Pacífico, a $670 \mathrm{~km}$ al oeste de Valparaíso, Chile, comprende las islas de Robinson Crusoe o Más a Tierra, Alejandro Selkirk o Más Afuera y el islote Santa Clara (LópezSepúlveda et al. 2015). La familia Poaceae comprende el $11 \%$ de la flora de estas islas y está representada por 32 géneros y 53 especies, con un alto porcentaje $(81,2 \%)$ de especies introducidas (Danton \& Perrier 2006, Baeza et al. 2002, 2007). Sólo dos géneros de gramíneas son endémicos del Archipiélago, Megalachne, con dos especies previamente descritas y Podophorus Phil., con una sola especie, P. bromoides Phil., la cual está extinta (Walter \& Gillert 1998).

Megalachne fue descrito por Steudel en 1854 con una sola especie ( $M$. berteroniana Steud.). Al describir el género, Steudel (1854) escribe en el protólogo "Bromus?" lo que ha sido interpretado tanto por Tateoka (1962) como por Matthei (1974) como una sugerencia sobre la afinidad de Megalachne con Bromus L.

Una segunda especie, M. zeylandica Thw., fue descrita en 1864 por Thwaites pero este taxón fue posteriormente transferido al género Eriachne (Lazarides 1995).

Philippi (1856) describe el género Pantathera con una especie, P. fernandezianana Phil. Posteriormente Hackel (1887) trata a Pantathera como sinónimo de Megalachne. Adicionalmente, este mismo autor diferencia los géneros Megalachne y Bromus por la presencia en Megalachne de 3 estigmas apicales y glumas aristuladas, mientras Bromus presenta 2 estigmas subapicales y glumas múticas.

Skottsberg \& Pilger (Pilger 1920), describen Bromus 
(Megalachne) masafueranus Skottsb. \& Pilg., donde relacionan a esta nueva especie con Megalachne y señalan que Megalachne debería estar incluida en la sección Ceratochloa de Bromus (Pilger 1920).

Skottsberg (1922) transfiere Pantathera fernandeziana al género Bromus, como B. fernandezianus. Más tarde, Pilger (1954) reconoce a Megalachne como un género diferente de Bromus, señalando para diferenciarlos, las mismas características que había propuesto Hackel (1887) e incluye la distribución geográfica como una característica importante, indicando que Megalachne se encuentra restringido al Archipiélago de Juan Fernández, mientras que Bromus tiene una distribución más amplia, estando presente en los continentes de ambos hemisferios.

Tateoka (1962) señala que a pesar de que Megalachne se ha relacionado con Bromus, en especial con los integrantes de la sección Ceratochloa porque comparten una serie de caracteres tales como las espiguillas comprimidas, las lemmas aquilladas y de márgenes hialinos, estas semejanzas no son tan importantes como sus diferencias y existe evidencia en la morfología del ovario, granos de almidón y serología que apoyan el tratamiento como dos géneros independientes y adicionalmente, indica que los caracteres florales, la pilosidad del ovario y la emergencia apical de los estilos acercan al género Megalachne a Festuca L. y no a Bromus.

Matthei (1974) concluye que Megalachne es un género diferente e independiente de Bromus, que se caracteriza porque los estilos nacen en el ápice del ovario, las lodículas son bilobadas, la pálea se encuentra adherida al fruto, las glumas son aristadas, la lígula está profundamente partida, la vaina es abierta y además, se trata de un género endémico de Juan Fernández, mientras que en Bromus los estilos nacen de la parte lateral del ovario, las lodículas son enteras, las glumas son múticas, la palea está fuertemente soldada al fruto, la lígula es entera o dentada y tiene una distribución más amplia que Megalachne. En este mismo trabajo, Matthei (1974) transfiere Bromus masafueranus al género Megalachne, estableciendo así que este género está formado por dos especies. Diferencia $M$. berteroniana Steud. de M. masafuerana (Skottsb. \& Pilger) Matthei por la longitud de las glumas aristadas que miden entre 33-35 mm, mientras que en $M$. masafuerana las glumas son aristuladas y menores a $25 \mathrm{~mm}$. Baeza et al. (2002) distinguen a ambas especies de Megalachne por la longitud de la arista de la lemma y la presencia y grado de desarrollo de las aristas de las glumas.

En consecuencia, Megalachne cuenta hasta ahora con 2 especies: $M$. berteroniana Steud. y $M$. masafuerana (Skottsb. \& Pilg.) Matthei, presentes en las islas Robinson Crusoe y Alejandro Selkirk, respectivamente.

Si bien los trabajos de Matthei (1974) y Baeza et al. (2002) clarifican y aportan a la taxonomía del género y sus especies, los caracteres utilizados como diagnósticos en ambos trabajos presentan una amplia variabilidad morfológica, siendo complejo establecer discontinuidades morfológicas entre ambas especies, en especial en los estados de caracteres de las glumas. Por otra parte, la revisión de las colecciones de herbario reveló la existencia de una tercera especie previamente confundida con $M$. berteroniana, la que se describe en este trabajo.

Danton \& Perrier (2006), al realizar el Nuevo Catálogo de las Plantas Vasculares del Archipiélago de Juan Fernández, señalan la posible existencia de una tercera especie en el género Megalachne, esta aseveración la realizan sobre la base de material recolectado en la isla Alejandro Selkirk.

En el presente trabajo se realiza una revisión del género Megalachne, se describe una nueva especie y se hace un análisis de los caracteres morfológicos y anatómicos mediante métodos multivariados, con el objeto de seleccionar los caracteres diagnósticos que mejor contribuyan al reconocimiento y diferenciación de los taxones.

\section{MATERIALES Y MÉTODOS}

\section{Morfología y AnATOMÍA}

Se analizaron 43 especímenes del género Megalachne depositados en los herbarios CONC y SGO (Thiers 2014). Las referencias al material examinado se encuentran citadas junto al tratamiento taxonómico de las especies. Las mediciones de las espiguillas fueron realizadas utilizando un ocular micrométrico, para las mediciones se utilizó el antecio inferior de la espiguilla basal de la panoja.

Para la anatomía foliar y epidermis abaxial se utilizó una porción de 0,5-1,0 cm de la zona media de la lámina foliar de la penúltima hoja de una innovación estéril, según la metodología propuesta por Metcalfe (1960). Para la epidermis, las muestras fueron rehidratadas en agua caliente por 5 min y se retiró la epidermis adaxial y el mesófilo con ayuda de un bisturí y luego se montó en glicerina-agua. Los cortes de lámina fueron efectuados a mano alzada. Las observaciones fueron realizadas en un microscopio Zeiss Axioscop. Los esquemas se realizaron con ayuda de una cámara clara adosada al microscopio. Para microscopía electrónica de barrido (MEB) las muestras se metalizaron con oro y observadas en un microscopio ETEC Autoscan en el Laboratorio de Microscopía Electrónica de la Universidad de Concepción. Para las descripciones de los apéndices epidérmicos y cortes transversales se siguió la terminología propuesta por Ellis $(1976,1979)$.

\section{ANÁLISIS ESTADÍSTICO MULTIVARIADO}

Se construyó una matriz básica de datos (MBD) con 23 OTUs y 26 caracteres (Apéndice I), 9 de los cuales fueron cualitativos (1 vegetativo, 8 reproductivos) y 17 cuantitativos (8 vegetativos, 9 reproductivos). A las variables cualitativas se aplicó la prueba de normalidad de 
Shapiro-Wilks modificada por Mahibbur \& Govindarajulu (1997). De las 17 variables cuantitativas 7 no cumplieron el supuesto de normalidad por lo que fueron transformadas a $\log _{10}$. La MBD fue estandarizada por filas y columnas antes del análisis. Se realizó un análisis estadístico descriptivo de los datos para determinar mínimo, máximo, media (o moda), desviación estándar y coeficiente de variación. La variación de los caracteres entre las tres especies detectadas se analizó mediante gráficos de caja y bigote. Las relaciones entre los especímenes fueron analizadas mediante análisis de coordenadas principales (ACoP), utilizando el índice de Gower que permite el uso de variables cualitativas y cuantitativas simultáneamente (Gower 1966). Se calculó el coeficiente de correlación cofenética, como medida de la distorsión producida por el análisis con respecto de la matriz original (MBD). Los análisis estadísticos se realizaron con el programa estadístico InfoStat versión 2013 (Di Rienzo et al. 2013). Los resultados de ACoP se graficaron con el programa Origin Lab.

\section{RESULTADOS}

ANÁLISIS ESTADÍsTICO

La variabilidad de los caracteres utilizados en el género Megalachne se muestra en la Tabla 1. A lo largo de la primera coordenada se separan M. berteroniana y M. masafuerana mientras Megalachne sp. se separa de las dos especies anteriores hacia el extremo positivo de la segunda coordenada. Los grupos resultantes son los siguientes (Fig. 1):

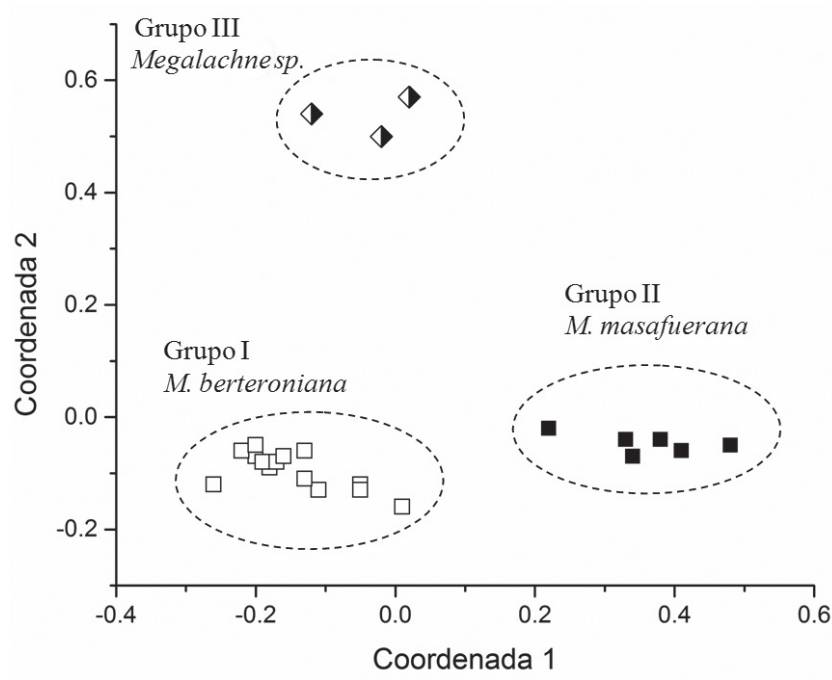

Figura 1. Distribución de las OTUs en los dos primeros componentes principales. $\mathrm{MB}=$ Megalachne berteroniana, $\mathrm{MM}$ $=$ M. masafuerana, Msp. = Megalachne sp. / Distribution of the OTUs in the two first principal components. $\mathrm{MB}=$ Megalachne berteroniana, $\mathrm{MM}=$ M. masafuerana, Msp. $=$ Megalachne $\mathrm{sp}$.
GRUPO I: Comprende 14 especímenes previamente determinados como $M$. berteroniana, caracterizados por poseer espiguillas de 3,4-7 cm de largo, con glumas largamente aristadas, lemmas aquilladas, escabrosas, cubiertas de ganchos, pero con punteaduras inconspicuas, con márgenes membranosos que alcanzan $1 / 3$ del ancho de la lemma en vista lateral, y callo con pelos cortos $(0,4-0,6$ $\mathrm{mm}$ de largo) (Fig. 2A). Los especímenes estudiados de este grupo provienen de la isla Robinson Crusoe, entre el nivel del mar y los 1.350 m s.n.m.

GRUPO II. Comprende 6 especímenes correspondientes a M. masafuerana. Se caracteriza por sus glumas notablemente más cortas que en el grupo anterior, con lemmas aquilladas, provistas de ganchos y punteaduras conspicuas, hispídulas, con márgenes membranosos que alcanzan $1 / 4$ del ancho de la lemma en vista lateral y pelos del callus cortos $(0,4-0,6$ mm de largo) (Fig. 2B). Los especímenes estudiados de este grupo provienen de la isla Alejandro Selkirk.

Grupo III. Comprende sólo 3 especímenes previamente confundidos con M. berteroniana, Bromus masafueranus y M. masafuerana. Los caracteres cuantitativos muestran una posición intermedia en relación con $M$. berteroniana y M. masafuerana, excepto para el largo de la espiguilla y antecios cuya media es mayor que en estas dos especies. Entre los caracteres cualitativos, uno de los más importantes es la forma de la lemma en sección transversal. En este grupo es redondeada mientras en $M$. berteroniana y $M$. masafuerana es aquillada, lateralmente comprimida. Los análisis permitieron además detectar que la lemma presenta pelos del callus más largos (1,4-1,6 mm v/s 0,4-0,6 mm en los otros dos grupos) y diferencias en la ornamentación de la lemma (Fig. 2 C). Considerando las diferencias establecidas, se reconoce a este grupo como una nueva especie que habita en la Isla Robinson Crusoe.

La variabilidad de los caracteres utilizados en el género Megalachne se muestra en la Tabla I. La distribución de las OTUs en los dos primeros componentes principales (PCoA), explican un $80 \%$ de la variabilidad de la matriz original (Fig. 1). A lo largo del primer componente se separa M. masafuerana hacia el extremo negativo del eje de $M$. berteroniana + Megalachne sp. Las variables que mejor explican la variación en el primer componente son largo de la gluma inferior, LG1 (vector propio 0,44), largo de la gluma superior, LG2 $(0,43)$ y largo del antecio, LANT $(0,41)$. Al segundo componente contribuyen, en mayor proporción, las variables número de nervios de la gluma superior, NG2 $(0,78)$ y relación largo arista/largo antecio, LA/LANT $(-0,45)$. La incorporación de variables cualitativas en análisis de coordenadas principales (PCoA) permite distinguir tres grupos de especies. La variabilidad de los caracteres cuantitativos en los tres grupos detectados se muestra en la Figura 3. 

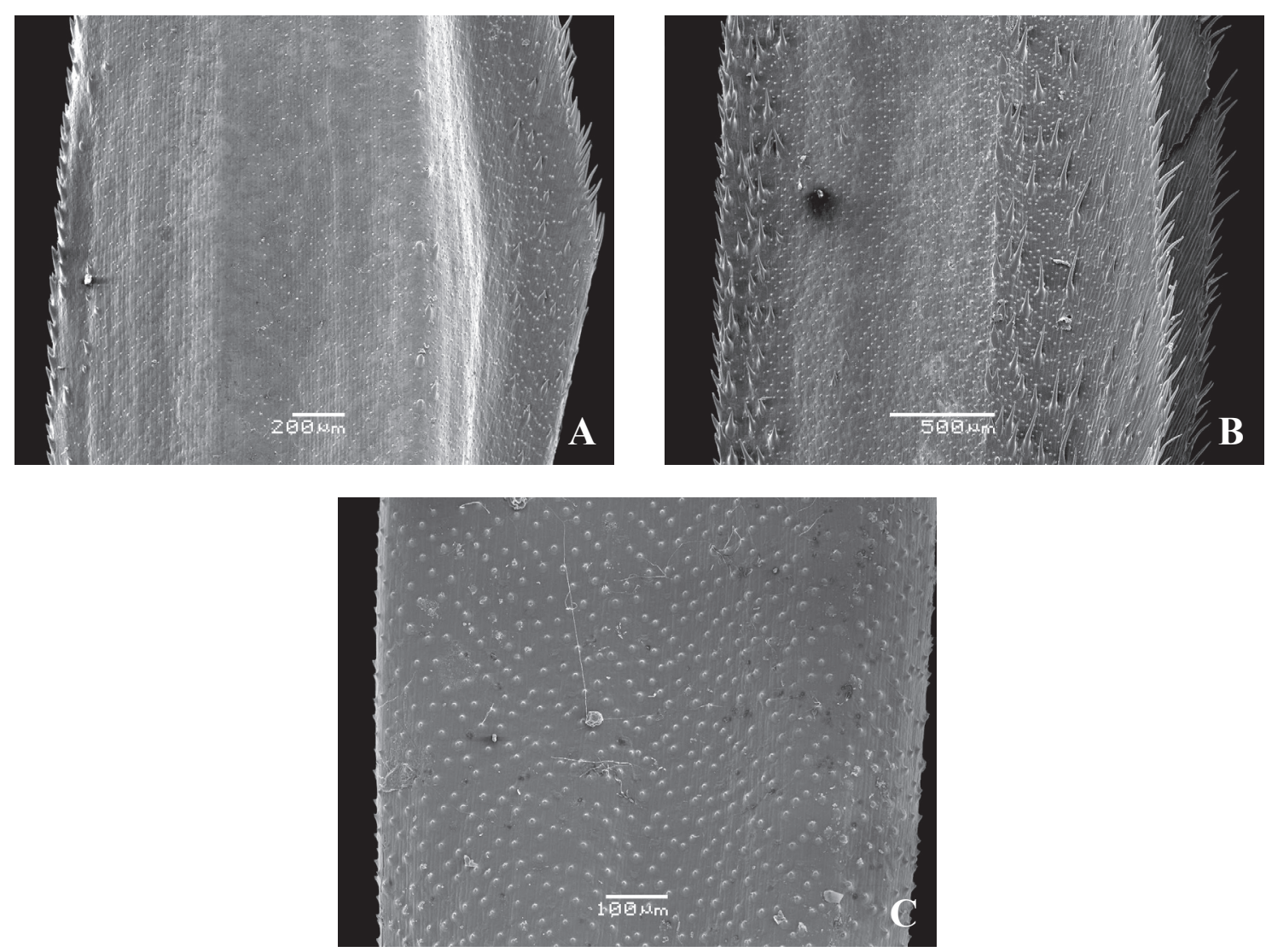

Figura 2. Fotomicrografía MEB de la superficie de la lemma. Detalle de los apéndices de la lemma. A: M. berteroniana (Sparre 292); B: M. masafuerana (C. Muñoz \& E. Sierra 7158); C: Megalachne sp. (C. Muñoz \& E. Sierra 7332). / SEM photomicrograph of the lemma surface. Details of the appendices of the lemma. A: M. berteroniana (Sparre 292); B: M. masafuerana (C. Muñoz \& E. Sierra 7158); C: Megalachne sp. (C. Muñoz \& E. Sierra 7332).

TABLA 1. Variación morfométrica de los caracteres utilizados en los análisis numéricos de Megalachne (Mín= valor mínimo; Máx = valor máximo; $\mathrm{DE}=$ desviación estándar y $\mathrm{CV}=$ coeficiente de variación). / Morphometric variation of the characters used in numerical analyses of Megalachne $(\mathrm{Min}=$ minimum value; $\mathrm{Max}=$ maximum value; $\mathrm{DE}=$ standard deviation; $\mathrm{CV}=$ coefficient of variation $)$.

\begin{tabular}{|c|c|c|c|c|c|c|c|}
\hline VARIABLE & ABREVIATURA & $\mathrm{n}$ & $\begin{array}{c}\text { Media o } \\
\text { Moda }\end{array}$ & D.E. & $\mathrm{CV}$ & Mín & MÁx \\
\hline \multicolumn{8}{|l|}{ VARIABLES CUALITATIVAS } \\
\hline 1 Forma de la lemma & FOLEM & 23 & 1 & - & - & 1 & 2 \\
\hline 2 Pelos del callus & PECAL & 23 & 1 & - & - & 1 & 2 \\
\hline 3 Tipo de apéndices de la lemma & APLEM & 23 & 1 & - & - & 1 & 3 \\
\hline 4 Forma cómo la lemma cubre a la pálea & LEPAL & 23 & 1 & - & - & 1 & 2 \\
\hline 5 Distribución de los apéndices de la lemma & DIALE & 23 & 1 & - & - & 1 & 3 \\
\hline 6 Ornamentación dorso de la lemma & ORDLE & 23 & 1 & - & - & 1 & 3 \\
\hline 7 Distribución de los apéndices del dorso de la lemma & DIADO & 22 & 1 & - & - & 1 & 2 \\
\hline 8 Ancho del margen membranáceo de la lemma & AMALE & 23 & 1 & - & - & 1 & 3 \\
\hline 9 Forma del nudo & FONUD & 17 & 1 & & - & 1 & 2 \\
\hline \multicolumn{8}{|l|}{ VARIABLES CUANTITATIVAS } \\
\hline 10 Largo de la gluma inferior $(\mathrm{cm})$ & LAGL1 & 23 & 2.18 & 0.79 & 36.15 & 1.00 & 3.90 \\
\hline 11 Número de nervios de la gluma inferior $\left(\mathrm{n}^{\circ}\right)$ & NEGL1 & 23 & 4.39 & 0.94 & 21.43 & 3.00 & 5.00 \\
\hline 12 Largo arista/largo total de la gluma inferior & LA/LG1 & 23 & 0.38 & 0.19 & 48.99 & 0.04 & 0.90 \\
\hline 13 Largo de la gluma superior $(\mathrm{cm})$ & LAGL2 & 23 & 2.36 & 0.89 & 37.77 & 1.10 & 4.10 \\
\hline
\end{tabular}




\begin{tabular}{lcrrrrrr}
\hline VARIABLE & ABREVIATURA & $\mathrm{n}$ & $\begin{array}{r}\text { MEDIA o } \\
\text { MoDA }\end{array}$ & D.E. & CV & Mín & MÁx \\
\hline 14 Número de nervios de la gluma superior $\left(\mathrm{n}^{\circ}\right)$ & NEGL2 & 23 & 4.57 & 0.84 & 18.48 & 3.00 & 5.00 \\
15 Largo arista/largo total de la gluma superior & LA/LG2 & 23 & 0.36 & 0.14 & 40.40 & 0.10 & 0.60 \\
16 Largo arista/largo antecio & LA/LAN & 23 & 0.66 & 0.10 & 15.79 & 0.50 & 0.80 \\
17 Largo del antecio (cm) & LANTE & 23 & 3.58 & 1.22 & 34.07 & 1.90 & 6.40 \\
18 Largo de la espiguilla (cm) & LESPI & 23 & 4.18 & 1.22 & 29.18 & 2.50 & 7.00 \\
19 Altura de la planta $(\mathrm{cm})$ & APLAN & 15 & 70.36 & 29.40 & 41.79 & 32.00 & 128.80 \\
20 Diámetro de la caña florífera $(\mathrm{cm})$ & DICFL & 16 & 0.79 & 0.30 & 37.22 & 0.50 & 1.70 \\
21 Largo de la vaina $(\mathrm{cm})$ & LAVAI & 17 & 5.28 & 1.37 & 25.87 & 3.20 & 7.20 \\
22 Largo de la lígula $(\mathrm{cm})$ & LALIG & 17 & 5.60 & 3.17 & 56.62 & 1.15 & 10.00 \\
23 Largo de la panícula $(\mathrm{cm})$ & LAPAN & 17 & 14.34 & 3.57 & 24.89 & 9.10 & 24.00 \\
24 Ancho de la panícula $(\mathrm{cm})$ & APANI & 16 & 7.58 & 2.36 & 31.13 & 4.10 & 11.50 \\
25 Largo de las láminas $(\mathrm{cm})$ & LALAM & 17 & 18.31 & 5.03 & 27.48 & 9.85 & 32.50 \\
26 Largo del entrenudo $(\mathrm{cm})$ & LAENU & 17 & 8.73 & 3.56 & 40.76 & 5.00 & 18.20 \\
\hline
\end{tabular}
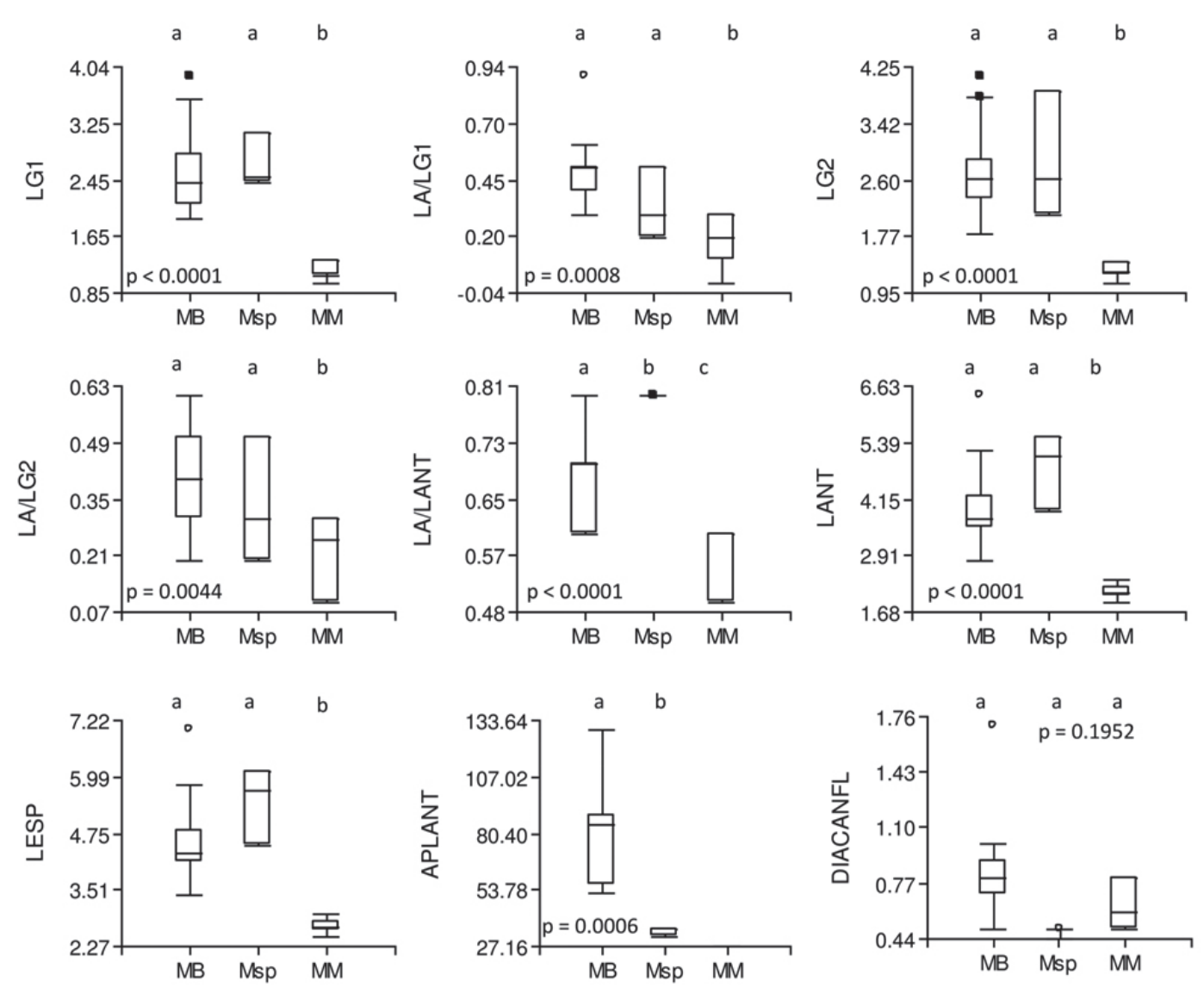

Figura 3. Variación de los nueve caracteres morfológicos cuantitativos en las tres especies de Megalachne. $\mathrm{MB}=$ M. berteroniana, MM = M. masafuerana, Msp. = Megalachne sp. Las cajas entregan los valores entre el primer y tercer cuartil; las líneas horizontales en el interior de las cajas señalan las medianas, las líneas verticales indican los rangos; los puntos negros indican los valores extremos y los círculos blancos indican los outliers. Letras distintas entre cajas indican diferencias estadísticas significativas. / Variation of nine morphological quantitative characters in the three species of Megalachne. $\mathrm{MB}=$ M. berteroniana, $\mathrm{MM}=$ M. masafuerana, Msp. $=$ Megalachne $\mathrm{sp}$. Boxes give values between the first and third quartiles; the medians are indicated by horizontal lines inside the boxes, the vertical lines indicate the ranges; black points are extreme values and white circles indicate outliers. Different letters among boxes indicate significant differences. 


\section{DISCUSIÓN}

Trabajos previos en el género Megalachne reconocieron sólo dos especies, M. berteroniana y M. masafuerana (Matthei 1974, Baeza et al. 2002). Estas dos especies están descritas sobre la base de caracteres de la espiguilla, cuya principal diferencia es la presencia de glumas aristadas o aristuladas, y la longitud de la arista de las lemmas. Si bien estos caracteres ayudan a la distinción de las especies, el límite entre aristado o aristulado es difuso. Además, los análisis numéricos muestran que al utilizar la longitud de las aristas de las glumas y de las lemmas, existen variaciones de este carácter dentro de los grupos que quedan definidos como $M$. berteroniana y $M$. masafuerana, ya que por ejemplo hay algunos ejemplares de $M$. berteroniana que presentan aristas muy largas en comparación con otros. Al incorporar un mayor número de caracteres, incluidos caracteres cualitativos, y de ejemplares y al analizarlos a través de métodos numéricos se pudo superar este problema y se agregaron nuevos caracteres diagnósticos además de los propuestos por Matthei (1974) y Baeza et al. (2002). Los análisis numéricos evidencian la existencia de una tercera entidad definida principalmente por la forma del antecio, la forma redondeada de la lemma, el tipo de apéndices y la distribución de estos en la superficie de la lemma, la forma en que la lemma cubre a la pálea y la longitud de los pelos del callo. Por lo tanto y de acuerdo a los resultados obtenidos, se considera que existe evidencia para distinguir en el género Megalachne tres especies, ya que hay suficientes caracteres que permiten diferenciar entre las entidades. Por otra parte, comparten otros que las hacen estar incluidas dentro del mismo género, como son los caracteres anatómicos foliares, los que prácticamente no muestran diferencias entre las tres entidades y que son un carácter útil para la delimitación de género; además, para las tres especies reconocibles para este género la región ITS no presenta variaciones (Peña 2007). Entre los caracteres morfológicos, la forma de la lemma, la longitud de las aristas de las glumas y de la lemma, la distribución de los apéndices en la superficie de esta última, son útiles para la delimitación de las especies. Basados en estos resultados, se describe una nueva especie $M$. robinsoniana $C$. Peña. Sin embargo, no se pudo corroborar si esta nueva especie es la misma a la que hacen referencia Danton \& Perrier (2006), ya que no fue posible tener acceso al material. Estos autores indican que la entidad que podría ser una nueva especie fue encontrada en la Isla Alejandro Selkirk (Masafuera), mientras que el material depositado en los herbarios CONC y SGO procede de la Isla Robinson Crusoe (Masatierra).

\section{TRATAMIENTO TAXONÓMICO}

Megalachne Steud., Syn. Pl. Glumac. 1: 237. 1854. Pantathera Phil., Bot. Zeitung (Berlin) (36): 649. 1856.
Plantas perennes, cespitosas, de 28-130 cm. Cañas cilíndricas, glabras, nudos engrosados o comprimidos. Vainas glabras, abiertas 3/4-2/3, menores o de igual longitud que el entrenudo; lígulas de $5-11 \mathrm{~mm}$, membranáceas, fuertemente partidas; láminas de 8-32 cm x 5-8 mm, planas, plegadas a convolutas. Inflorescencias en panículas laxas, de 8,5-23 x 4-12 cm. Espiguillas comprimidas lateralmente, de (2,5)3,5-7 cm, 3-6-floras, desarticuladas sobre las glumas y entre los antecios; raquilla prolongada más allá del antecio distal; glumas subiguales, la superior levemente más larga que la inferior, de igual tamaño que los antecios adyacentes, membranáceas, lanceoladas, aristuladas o aristadas, aristas de las glumas más cortas que la de las lemmas, (3-4)-5-nervias; lemmas lanceoladas a obovadas, subcoriáceas, aquilladas o redondeadas, 5 nervias, muriculadas y/o con ganchos o aguijones; callo obtuso, piloso, pelos de 0,4-1,6 mm; arista apical, persistente, escabriúscula, no geniculada, tanto o más larga que el cuerpo de la lemma; pálea elíptica, de 8-10 mm x 1-1,5 mm, levemente menor, o igual que la lemma; carinas conspicuas, con aguijones, ápice bidentado; lodículas 2, libres, membranáceas, bilobuladas. Anteras fértiles de 4-6 $\mathrm{mm}$, estaminodios de 0,6-2 mm; ovario piloso en el ápice, estigmas 2-3, apicales, libres o fusionados basalmente; antecios perfectos e imperfectos. Cariopsis comprimida dorsiventralmente, 4-7 mm de largo, con el ápice piloso; hilo linear, tan largo como el cariopsis.

Género con tres especies endémicas del Archipiélago de Juan Fernández, afín a Bromus y Festuca. Soreng (2003) sugirió que pertenecía a la tribu Stipeae subtribu Duthieinae, pero estudios filogenéticos basados en datos moleculares han demostrado que debe ser incluido en la tribu Poeae (Romaschenko et al. 2012), subtribu Loliibae (Soreng et al. 2015).

ESPECIE TIPO: Megalachne berteroniana Steud.

EPIDERMIS Y ANATOMÍA FOLIAR

Sección transversal de la lámina: Contorno de la lámina plana o convoluta; haces vasculares de primer orden trabados, con bandas de tejido esclerenquimático conectadas a ambas epidermis; banda continua de tejido esclerenquimático ausente en ambas epidermis; células buliformes y macropelos presentes sólo en la epidermis adaxial (Fig. 4F).

Epidermis abaxial: Zonas costales e intercostales poco definidas. Células largas, intercostales rectangulares con los extremos redondeados de paredes onduladas; macropelos ausentes; papilas ausentes; estomas poco frecuentes cercanos a las costillas; células de corcho en forma de media luna, las de sílice redondeadas; células largas de las costillas onduladas; las células de sílice de igual forma que en las zonas intercostales; aguijones ausentes; las paredes de las células de las costillas y de las zonas intercostales presentan el mismo grosor. Las 
diferencias en los caracteres cualitativos y cuantitativos entre las tres especies determinadas se muestran en las Tablas 2 y 3.
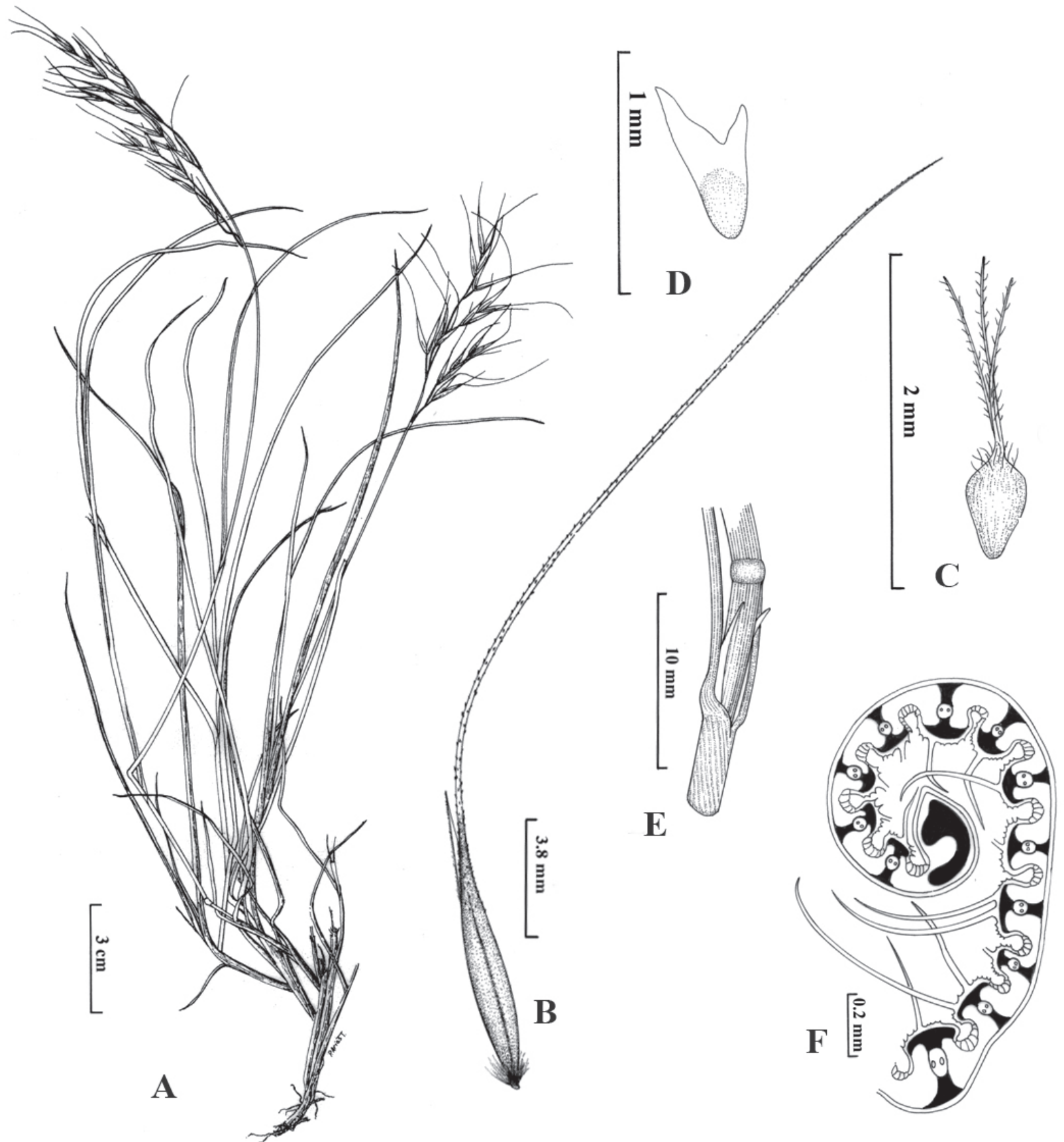

DistribuCIÓN GEOGRÁFICA

Género endémico del Archipiélago de Juan Fernández, Chile (Fig. 5). M. masafuerana está presente en A. Selkirk, mientas que $M$. berteroniana y $M$. robinsoniana crecen en Robinson Crusoe, sobre roquedales y en quebradas, tanto fuera como dentro del bosque, entre los 5 y los 1.100 m s.n.m.

Figura 4. A-C. Megalachne robinsoniana. A. Planta; B. Antecio; C. Ovario; D. Lodícula; E. Lígula (A-E tipo, Faúndez s.n.); M. masafuerana F. Corte transversal de la lámina (Muñoz \& Sierra 7332). / A-C. Megalachne robinsoniana. A. Plant; B. floret; C. ovary; D. lodicule; E. ligule (A-E, Faúndez s.n.). M. masafuerana F.Leaf blade transection (Muñoz \& Sierra 7332). 


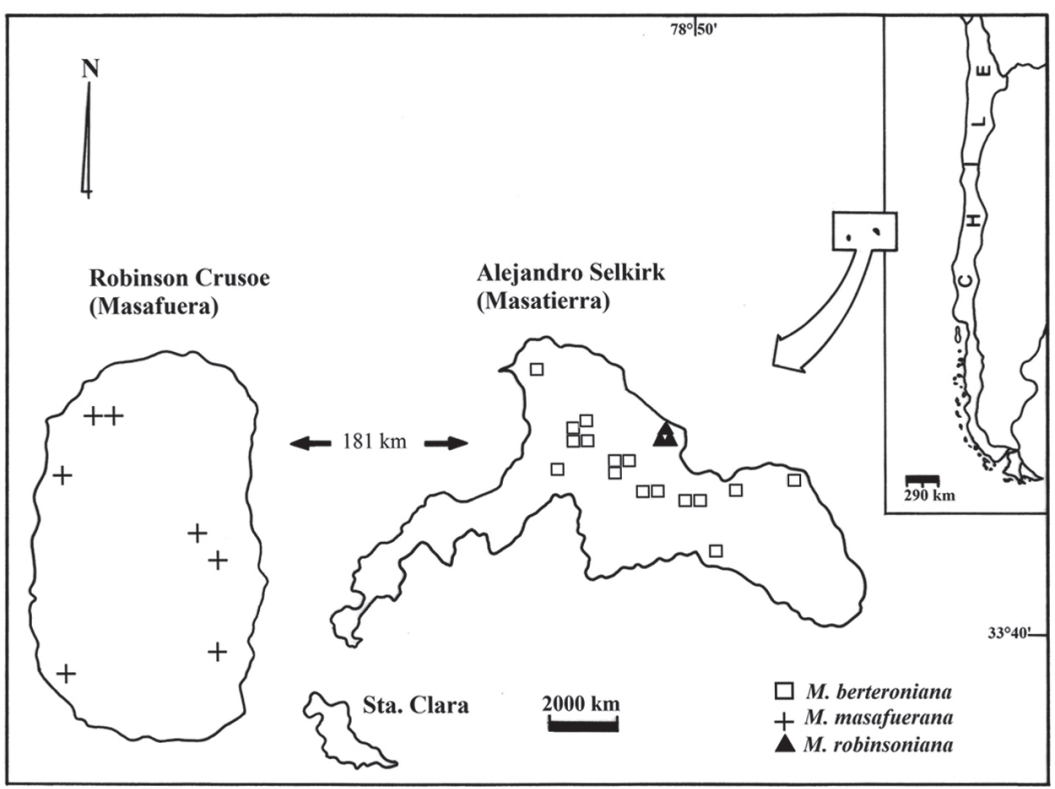

Figura 5. Mapa de distribución de las especies de Megalachne en el archipiélago de Juan Fernández. / Distribution of the species of Megalachne in the Juan Fernández Archipelago.

Tabla 2. Variación de los caracteres cuantitativos continuos en Megalachne berteroniana, M. masafuerana y M. robinsoniana. I Morphometric variation of the continuous quantitative characters in Megalachne berteroniana, M. masafuerana and M. robinsoniana.

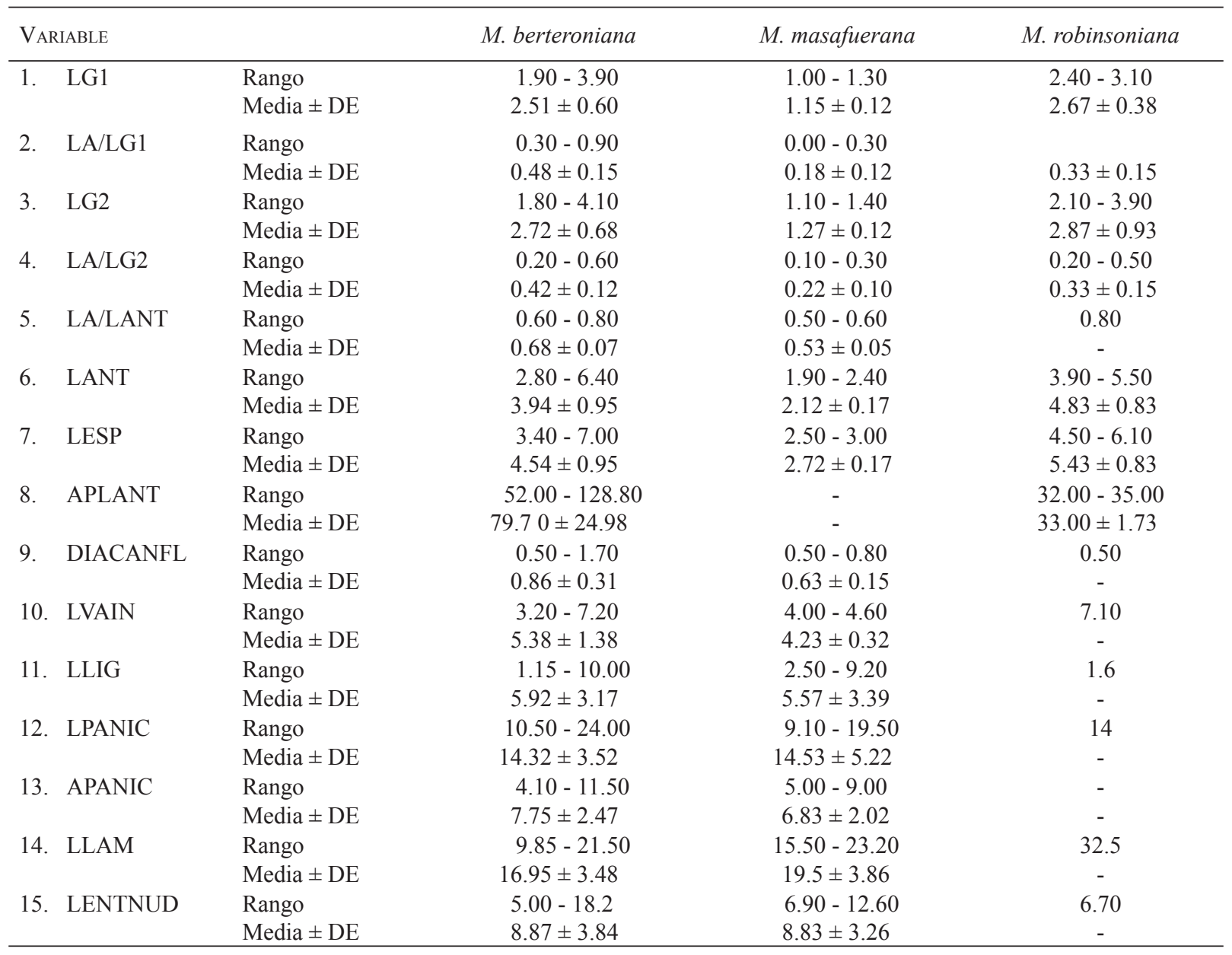


Megalachne (Poaceae) en Juan Fernández: PeÑA, C. ET AL.

TABLA 3. Variación de los caracteres cuantitativos discretos y cualitativos en Megalachne berteroniana, M. masafuerana y M. robinsoniana. / Morphometric variation of the discrete quantitative and qualitative characters in Megalachne berteroniana, M. masafuerana and M. robinsoniana.

\begin{tabular}{|c|c|c|c|c|}
\hline $\mathrm{N}^{\circ}$ & CARÁCTER / TAXON & M. berteroniana & M. masafuerana & M. robinsoniana \\
\hline 2 & $\mathrm{~N}^{\circ}$ nervios gluma inferior & $3-5$ & $3-5$ & 5 \\
\hline 5 & $\mathrm{~N}^{\circ}$ nervios gluma superior & 5 & $3-5$ & $3-5$ \\
\hline 7 & Sección transversal de lemma & Aquillada & Aquillada & Redondeada \\
\hline 8 & Largo pelos del callo (mm) & $0,4-0,6$ & $0,4-0,6$ & $1,4-1,6$ \\
\hline 12 & Apéndices de la lemma & $\begin{array}{l}\text { Punteaduras inconspicuas y } \\
\text { ganchos }\end{array}$ & $\begin{array}{l}\text { Punteaduras conspicuas y } \\
\text { ganchos }\end{array}$ & $\begin{array}{l}\text { punteaduras conspicuas y } \\
\text { ganchos o sólo ganchos }\end{array}$ \\
\hline 13 & Margen de la lemma / pálea & $\begin{array}{l}\text { Pálea cubierta por los } \\
\text { márgenes de la lemma }\end{array}$ & $\begin{array}{l}\text { Pálea cubierta por los } \\
\text { márgenes de la lemma }\end{array}$ & $\begin{array}{l}\text { Lemma envuelve a la } \\
\text { pálea }\end{array}$ \\
\hline 14 & $\begin{array}{l}\text { Distribución de los apéndices en } \\
\text { la lemma }\end{array}$ & $\begin{array}{l}\text { Ganchos en el margen, en el } \\
\text { dorso y sobre los } 5 \text { nervios }\end{array}$ & $\begin{array}{c}\text { Ganchos en el margen y en } \\
\text { el dorso pero no sobre los } \\
\text { nervios }\end{array}$ & $\begin{array}{l}\text { Ganchos distribuidos } \\
\text { sobre la superficie }\end{array}$ \\
\hline 15 & $\begin{array}{l}\text { Ornamentación del dorso de la } \\
\text { lemma }\end{array}$ & Escabroso & Hispídulo & Hispídulo \\
\hline 16 & $\begin{array}{l}\text { Distribución de los apéndices en } \\
\text { el dorso }\end{array}$ & Desde el callo a la arista & Desde el callo a la arista & Desde la mitad superior \\
\hline 17 & $\begin{array}{l}\text { Ancho del margen membranáceo } \\
\text { de la lemma }\end{array}$ & $1 / 3$ del ancho de la lemma & $\begin{array}{c}\text { Hasta } 1 / 4 \text { del ancho de la } \\
\text { lemma }\end{array}$ & $\begin{array}{c}\text { Hasta } 1 / 5 \text { del ancho de la } \\
\text { lemma }\end{array}$ \\
\hline
\end{tabular}

Clave para la determinación de las especies de Megalachne

1. Plantas de 52 a $130 \mathrm{~cm}$. Lemmas aquilladas, plegadas en sección transversal, muriculadas y/o con ganchos distribuidos sobre los márgenes o los nervios de la lemma, márgenes membranáceos $>1 / 5$ del ancho total de la lemma, no envuelven a la pálea. Pelos del callo $<0,6 \mathrm{~mm}$

2. Lemmas inconspicuamente muriculadas, ganchos sobre el nervio medio y los nervios laterales. Margen membranáceo de $1 / 3$ del ancho total de la lemma. Glumas con aristas de aproximadamente 1/3 del largo total de la gluma. M. berteroniana 2'. Lemmas conspicuamente muriculadas, ganchos sólo sobre el nervio medio y en los márgenes. Margen membranáceo de 1/4 del ancho total de la lemma. Glumas con aristas de aproximadamente $1 / 5$ del largo total de la gluma..... M. masafuerana 1'. Plantas generalmente $<$ de $30 \mathrm{~cm}$. Lemmas redondeadas en sección transversal, convolutas, muriculadas y/o con ganchos en toda la superficie de la lemma, márgenes membranáceos, de 1/5 del ancho total de la lemma, envolviendo la pálea. Pelos del callo > de 1,4 mm M. robinsoniana

1. Megalachne berteroniana Steud., Syn. Pl. Glumac. 1: 237. 1854.

Tipo: Chile: Juan Fernández Islands, Jan-Jun 1830, Bertero 1440 (Holotipo: P, foto SGO 073157!; Isotipo: US 91419 (fragmento)).

Pantathera fernandeziana Phil., Bot. Zeitung (Berlin) 14 Tipo: Chile: Juan Fernández Islands, Oct 1859, F. Germain s.n. (Holotipo: SGO 63540!; Isotipo: B, SGO PHIL 476, US 91420, 91419 (fragmento). Pantathera avenacea Phil. ex Hemsl., Rep. Sci. Result Voyage H.M.S. Challenger, Bot. 1(3): 61.1884 (nom. nudum). Tipo: Chile, Juan Fernández Isls., Moselley s.n. (K). Bromus fernandezianus (Phil.) Skottsb., Nat. Hist. Juan Fernandez 2: 102, f. 1i. 1921.

Plantas de 52-130 cm; cañas glabras, nudos generalmente engrosados, del mismo color de la caña. Vainas abiertas, glabras; lígulas membranáceas, fuertemente partidas; láminas planas, de 9,8-21 cm x 5-8 mm, la cara adaxial pilosa. Espiguillas de 4,5-5,8 cm, 5-6 floras, flores hermafroditas, el antecio distal estéril; glumas persistentes, aristadas, (34)-5 nervias, gluma inferior de 1,9-3,9 cm; gluma superior de 1,8-3,8 cm; aristas de hasta $1 / 3$ del largo total de la gluma; lemmas lanceoladas, conduplicadas, subcoriáceas, aquilladas, 5-nervias, muriculadas con ganchos y/o aguijones en los nervios; arista generalmente curvada, persistente y escabriúscula; callo obtuso y piloso, pelos de $0,4-0,6 \mathrm{~mm}$ de longitud; pálea de igual consistencia que la lemma, levemente menor o de igual tamaño, biaquillada, con aguijones en las quillas, ápice bidentado; lodículas 2, libres, membranáceas, bilobuladas; ovario piloso en el ápice, estigmas 2-3, apicales. Cariopsis de $47 \mathrm{~mm}$ de longitud, con el ápice piloso. 
Material adicional estudiado: CHILE. Región de Valparaíso, Archipiélago de Juan Fernández, Isla Robinson Crusoe (Masatierra), Plazuela Yunque, oct 1934, C Bock s.n. (CONC 20333); 300 m, oct 1934, C. Bock s.n. (CONC 24430); Quebrada de la Damajuana, sobre rocas, $400 \mathrm{~m}$ s.n.m, 3 mar 1955, Sparre 292 (CONC 18674); Cerro Salsipuedes, 1350 m, 10 dic 1965, F.G. Meyer 9508 (CONC 40599); camino a cerro S de Damajuana, 21 nov 1980, Stuessy et al. 5351 (CONC 52977); Piedra Agujereada, 270 m, 22 nov 1980, Stuessy et al. 5390 (CONC 53014); Path toward Mirador de Selkirk up from San Juan Bautista, ca 500 m, 24 nov 1980 (CONC 53047); Mirador de Selkirk, 400 m s.n.m, 14 dic 1965, C. Muñoz \& E Sierra 7313 (CONC 69582); Quebrada El Pangal, 200 m, 12 dic 1965, C. Muñoz \& E. Sierra 7271 (CONC 69581); Cerro Alto, 3338/7852, 340 m, 21 oct 1966, A. Angulo 55 (CONC 43883); entre Bahía Cumberland y Puerto Inglés, 180 m, 31 oct 1966, A. Angulo 56 (CONC 43882); Valle Inglés hacia Cerro Agudo, 420 m, 12 feb 1990, Stuessy et al. 11630 (CONC 116540); Corrales de Molina, 340 m, 24 ene 1990, D. Wiens et al. 11203 (CONC 117010); 3338/7851, ene 1957, J.P. Simon s.n. (CONC 124942); sendero de Mirador Alejandro Selkirk a sector Villagra, 3338/7851, 464 m, 13 dic 2003, M.F. Gardner et al. 54 (CONC 162435); in the silla between the Cerro up from Mirador (toward Piramide), and Piramide, then down into the quebrada on NW side, epiphytic on Drimys, 670 m, 31 ene 1990, Baeza \& Peñailillo 11316 (CONC 116618).

2. Megalachne masafuerana (Skottsb. \& Pilg. ex. Pilg.) Matthei, Bol. Soc. Biol. Concepción 48: 171. 1974.

Basónimo: Bromus masafueranus Skottsb. et Pilg. ex. Pilg., Repert. Spec. Nov. Regni Veg. 16: 385. 1920. Tipo: Chile, Juan Fernández: Masafuera, "Hochland bei Las Torres, sterile Felsen" 1370 m, Feb 1917. C. Skottsberg \& I. Skottsberg 415 (Holotipo: GB; Isotipo: P, S, UPS, US8655446 (fragmento ex P)).

Plantas de 57-90 cm de altura. Cañas glabras, nudos engrosados, del mismo color de la caña. Vainas abiertas, glabras; lígulas membranáceas, fuertemente partidas; láminas planas de $8-32 \mathrm{~cm}$, la cara adaxial pilosa. Espiguillas 4-6 floras, el antecio distal estéril, de 2,1-4,1 $\mathrm{cm}$; glumas persistentes, aristuladas, (3-4)-5 nervias; gluma inferior de 1,0-2,4 cm; gluma superior de 1,1-2,5 cm, aristas de aproximadamente 1/5 del largo total de la gluma; lemmas lanceoladas, subcoriáceas, aquilladas, 5-nervias, conspicuamente muriculadas, con ganchos y/o aguijones distribuidos en el margen de la lemma y en el nervio dorsal; arista curvada, persistente y escabriúscula; la lemma cubre a la pálea entre sus márgenes; callo obtuso y piloso, pelos de 0,4-0,5 $\mathrm{mm}$ de longitud; pálea de igual consistencia que la lemma, levemente menor o igual, biaquillada, con ganchos en las quillas, ápice bidentado; lodículas 2, libres, membranáceas, bilobuladas; ovario piloso en el ápice, estigmas 2(3), apicales. Cariopsis, 3-6 mm de longitud, con el ápice piloso.

Material adicional estudiado: CHILE. Región de Valparaíso, Archipiélago de Juan Fernández, isla Robinson Crusoe, 3338/7852, $400 \mathrm{~m}$, feb 1937, J. Miranda s.n. (CONC 70220); Up trailfrom Plazoleta El Yunque to El Camote; El Camote on cliffs over the ocean, $570 \mathrm{~m}$, Stuessy et al. 11166 (CONC 116624); La Vaquería, $400 \mathrm{~m}, 33^{\circ} 38^{\prime} \mathrm{S}$, 7852’W, 7 abr 1983, B. López 46 (CONC 55459); Cordón Salsipuedes, $500 \mathrm{~m}$, on ridge with Ugni molinae on rocks, 15 ene 1991, Stuessy et al. 11751 (CONC 121444);Isla Alejandro Selkirk, 500 m, dic 1936, Barrientos s.n. (CONC 70221); Quebrada de La Colonia, 3345/8046, 500 m, 28 nov 1965, C. Muñoz \& E. Sierra 7123 (CONC 69580); 500 m, 30 nov 1965, C. Muñoz \& E. Sierra 7158 (CONC 69578); Quebrada Angosta, 1 dic 1965, F.G. Meyer 9405 (CONC 40597); Masafuera, Cuchillo del Imán, al norte de la isla, bajando de Cerro Verde, 880 m, ene-feb 1986, A Landero \& L. Gaete 9150 (CONC 111588); Quebrada Tongo, on south side down from the south ridge of Cordon Inocentes, $1100 \mathrm{~m}, 9$ feb 1986, H. Valdebenito 9660 (CONC 112177); Quebrada Vacas, 320 m, 28 ene 1986, Valdebenito \& Lammers 8193 (CONC 112377); Plano de Rodríguez, above the sea at Punta Buque Varado also known as Avenida de Las Cabras, 1090 m, 18 ene 1986, Stuessy et al. 9102 (CONC 112475); Quebrada Larga, 700 m, 5 feb 1986, Ruiz et al. 8312 (CONC 112482); Quebrada Casas, moist deep ravine, 150 m, 5 feb 1990, Baeza \& Peñailillo 11439 (CONC 116617); Quebrada Casas, upper limits on steep slopes, descending from ridge above, $810 \mathrm{~m}, 17$ ene 1986, Stuessy et al. 9074 (CONC 111857).

3. Megalachne robinsoniana C. Peña, sp. nov.

Tipo: Chile, Archipiélago Juan Fernández, Isla Robinson Crusoe, Puerto Inglés. Acantilado bajo, 5-30 m s.n.m., Faúndez s.n.3-I-1992 (Holotipo: SGO 122921) (Fig. 4 A-E).

Lemma obovatus, convolute, ad dorsum rotundatis, apice longe aristatum,subcoriaceum, puncticulatus et uncatus, callo puberulus 1,4-1,6 mm.

Plantas de $28-40 \mathrm{~cm}$ de altura. Cañas glabras, nudos comprimidos, castaño oscuro a negro. Vainas abiertas, glabras; lígulas membranáceas, fuertemente partidas; láminas generalmente lineares, de 9-32,5 cm, cara adaxial pilosa. Espiguillas 3-4 floras, el antecio distal estéril, de 4,1-7 cm long; glumas persistentes, aristadas, (3-4)-5 nervias, gluma inferior de 2,4-3,1 cm, con aristas de 5-18 $\mathrm{mm}$, gluma superior de 2,1-3,9 cm; lemmas obovadas, subcoriáceas, redondeadas, 5-nervias, muriculada y/o con ganchos distribuidos en la superficie de la lemma; arista curvada, persistente y escabriúscula; márgenes de 
la lemma convolutos; callo obtuso y piloso, pelos de 1-1,5 $\mathrm{mm}$ de longitud; pálea de igual consistencia que la lemma, levemente menor o igual, con aguijones en las quillas, ápice bidentado; lódiculas 2, libres, membranáceas, bilobuladas. Ovario piloso en el ápice; estigmas 3, apicales.

Material adicional estudiado: CHILE. Región de Valparaíso, Archipiélago de Juan Fernández, Isla Robinson Crusoe (Masatierra), Quebrada el Inglés, desde la orilla del mar hasta los \pm 400 m s.n.m., Muñoz \& Sierra 7332 (CONC 69579); south side of cerro Alto, above Puerto Inglés, 1000 ft., 14-XII-1965, F.G. Meyer 8567 (CONC 40598).

\section{AGRADECIMIENTOS}

Agradecemos a los Drs. Robert J. Soreng y Kanchi Gandhi la discusión de aspectos nomenclaturales, en especial la correcta ortografía de Megalachne berteroniana. A los correctores anónimos por sus sugerencias. A Alicia Marticorena, editora de Gayana Botánica.

\section{BIBLIOGRAFÍA}

Baeza, C.M., Stuessy, T., Marticorena, C. 2002. Notes on the Poaceae of the Robinson Crusoe (Juan Fernández) Islands, Chile. Brittonia 54(3): 154-163.

Baeza, C.M., Marticorena, C., Stuessy, T., Ruiz, E., Negritto, M.A. 2007. Poaceae de Juan Fernández (Robinson Crusoe). Gayana Botánica 64(2): 125-174.

Danton, P., Perrier, C. 2006. Nouveau catalogue de la flore vasculaire de l'archipel Juan Fernández (Chili). Acta Botanica Gallica 153(4): 399-587.

Di Rienzo J.A., Casanoves, F., Balzarini, M.G., González, L., Tablada, M., Robledo, C.W., InfoStat versión 2013. Grupo InfoStat, FCA, Universidad Nacional de Córdoba, Argentina.

ELLIS, R.P. 1976. A procedure for standardizing comparative leaf anatomy in the Poaceae. I. The leaf blade as viewed in transverse section. Bothalia 12(1): 65-109.

Ellis, R.P. 1979. A procedure for standardizing comparative leaf anatomy in the Poaceae. II. The epidermis as seen in surface view. Bothalia 12(4): 641-671.

Gower, J.C. 1966. Some distance properties of latent root and vector methods used in multivariate analysis. Biometrika 53: 325-338.

Hackel, E. 1887. Gramineae. In: Engler, A., Prantl, K. (eds.), Die natürlichen Pflanzefamilien, Vol. II, 2: 2-97.

LAZArides, M. 1995. The genus Eriachne (Eriachneae, Poaceae). Australian Systematic Botany 8(3): 355-342.
López-Sepúlveda, P., Takayama, K., Greimler, J., Crawford, D.J., Peñailillo, P., Baeza, M., Ruiz, E., Kohl, G., Tremetsberger, K., Gatica, A., Letelier, L., Novoa, P., NovaK, J., STuessy, T.F. 2015. Progressive migration and anagenesis in Drimys confertifolia of the Juan Fernández Archipiélago, Chile. Journal Plant Research 128: 73-90.

Matthei, O. 1974. El género Megalachne Steud. (Gramineae). Boletín de la Sociedad de Biología de Concepción 48: 165-172.

Mahibbur, R.M., Govindarajulu, Z. 1997. A modification of the test of Shapiro and Wilks for normality. Journal of Applied Statistics 24(2): 219-235.

Metcalfe, C.R. 1960. Anatomy of the Monocotyledons. I. Gramineae. Oxford University Press, London. 731 pp.

Peña, C. 2007. Posición tribal de Megalachne Steud. (Poaceae), basada en caracteres morfológicos y moleculares. Tesis. Magíster en Ciencias mención Botánica. Universidad de Concepción, Concepción, Chile. 75 pp.

Philippi, R.A. 1856. Observaciones sobre la flora de Juan Fernández. Anales de la Universidad de Chile 13: 157-169.

PIlger, R. 1920. Ueber einige Gramineae der Skottbergschen sammlung von Juan Fernández. Repertorium Specierum Novarum Regni Vegetabilis 16: 385-388.

Pilger, R. 1954. Das System der Gramineae. Botanische Jahrbücherfur Systematik 76: 310-311.

Romaschenko, K., Peterson, P.M., Soreng, R.J., García-Jacas, N., Futorna, O., Susanna, A. 2012. Systematics and evolution of the needle grasses (Poaceae: Pooideae: Stipeae) based on analysis of multiple chloroplast loci, ITS and lemma micromorphology. Taxon 61: 18-44.

Steudel, E.G. 1854. Syn. Pl. Glum. Stuttgart. 2 vols Pars I. Gramineae. 474 pp.

Soreng, R.J. 2003. Megalachne. In: Soreng, R.J., Davidse, G., Peterson, P.M., Zuloaga, F.O., Judziewicz, E.J., Filgueiras, T.S., Morrone, O. (eds.). Catalogue of New World Grasses (Poaceae): IV. Subfamily Pooideae. Contribution of the U.S. National Herbarium 46: 432.

Soreng, R.J., Peterson, P.M., Romaschenko, K., Davidse, G., Zuloaga, F.O., Judziewicz, E.J., Filgueiras, T.S., Davis, J.I., Morrone, O. 2015. A worldwide phylogenetic classification of the Poaceae. Journal of Systematics and Evolution 53: 117-137.

TAтeoka T. 1962. Starch grains of the endosperm in grass systematics. The Botanical Magazine (Tokyo) 75: 377-383.

Thiers, B. 2014 [continuously updated]. Index Herbariorum A global directory of public herbaria and associated staff. New York Botanical Garden's Virtual Herbarium. http:// sweetgum.nybg.org/ih/. Accesado: 14 marzo 2014.

Walter, K.S. \& H.J. Gillertt (eds). 1998. 1997 IUCN: Red List of Threatened Plants. Compiled by the World Conservation Union, Gland, Switzerland and Cambridge, UK. lxiv +862 pp.

Recibido: 31.07.2014

Aceptado: 04.04.2017 